\title{
Expression of Chicken NK-Lysin and Its Role in Chicken Coccidiosis Induced by Eimeria necatrix
}

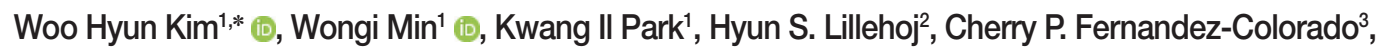 \\ Rochelle A. Flores ${ }^{1}$, Paula Leona T. Cammayo ${ }^{1}$, Binh Thanh Nguyen ${ }^{1}$ (D) \\ ${ }^{1}$ College of Veterinary Medicine \& Institute of Animal Medicine, Gyeongsang National University, Jinju 52828, Korea; ${ }^{2}$ Animal Biosciences and \\ Biotechnology Laboratory, Beltsville Agricultural Research Center, ARS, U.S. Department of Agriculture, Beltsville, MD 20705, USA; ${ }^{3}$ Department of \\ Veterinary Paraclinical Sciences, College of Veterinary Medicine, University of the Philippines Los Baños, College, Laguna 4031, Philippines
}

\begin{abstract}
Coccidiosis in chickens is an intestinal parasitic disease caused by protozoan parasites named Eimeria spp. In some Eimeria infections, intestinal lymphocytes are known to highly express chicken NK-lysin (cNK-lysin), an antimicrobial peptide with anticoccidial activity. Therefore, this study aims to investigate the expression of cNK-lysin in E. necatrix-infected chickens and its role in $E$. necatrix infection. The expression of cNK-lysin transcript was significantly increased in $E$. necatrix sporozoites-treated lymphocytes. In E. necatrix infection, cNK-lysin transcript was induced in intestinal lymphocytes but not in the spleen. The recombinant cNK-lysin exhibited anticoccidial activity against $E$. necatrix sporozoites as well as immunomodulatory activity on macrophages by inducing proinflammatory cytokines. These results indicated that E. necatrix infection induces high local expression of cNK-lysin and the secreted cNK-lysin helps protect coccidiosis.
\end{abstract}

Key words: Eimeria necatrix, chicken, NK-lysin, antimicrobial peptide, coccidiosis

\section{INTRODUCTION}

Chicken coccidiosis is an intestinal parasitic disease caused by intracellular protozoan parasites, Eimeria spp. that belongs to the phylum Apicomplexa. Eimeria spp., have a single host, and there are 7 species of Eimeria, E. acervulina, E. maxima, E. tenella, E. necatrix, E. necatrix, E. praecox, and E. mitis, which have known to cause chicken coccidiosis [1]. Among these, coccidiosis by E. acervulina, E. maxima, and E. tenella are found most often in poultry worldwide thus, they are considered as major species in chicken coccidiosis. Eimeria spp. are highly site-specific and E. necatrix infects the mid-intestinal area with hemorrhage resulting in a high mortality rate [2]. As coccidiosis in chickens characterized by multiple species infection, it is needed to study non-major species for developing a control strategy to mitigate coccidiosis in chickens [3]. They have complex life cycle which is consist of sexual/asexual and intra/extracellular developmental stages. The sporozoites, motile and

\footnotetext{
- Received 10 August 2021, revised 10 October 2021, accepted 11 October 2021.

*Corresponding author (Woohyun.Kim@gnu.ac.kr)

(C) 2021, Korean Society for Parasitology and Tropical Medicine

This is an Open Access article distributed under the terms of the Creative Commons Attribution Non-Commercial License (https://creativecommons.org/licenses/by-nc/4.0) which permits unrestricted non-commercial use, distribution, and reproduction in any medium, provided the original work is properly cited.
}

infective form of parasite, are released from sporocysts in the intestinal lumen, and invade the intestinal linings to develop inside the cells. Many studies have focused on the sporozoites antigen because they are being contacted with host cells and it is recognized as the beginning of the infection $[4,5]$.

Chicken NK-lysin (cNK-lysin), a homolog of human granulysin is an antimicrobial peptide (AMP) produced by cytotoxic $\mathrm{T}$ and natural killer (NK) cells, and it is identified that cNK-lysin is highly expressed in Eimeria-infected intestinal lymphocytes [6-8]. It has also been demonstrated that cNK-lysin have an anticoccidial effect against some Eimeria spp. including E. acervulina, E. maxima, and E. tenella and this activity of cNK-lysin lead to evaluation as a candidate of anticoccidial target $[7,9]$. Moreover, subsequent studies have shown that cNK-2, a core region of $\mathrm{cNK}-l y s i n$ showing higher antimicrobial activity against Eimeria spp., modulates immune responses in chicken macrophages and that the administration of $\mathrm{cNK}-2$ in E. acervulina infection reduced intestinal pathology [10,11]. There are increasing studies on AMPs that possess a dual action mechanism which are direct killing activity against pathogens and immunomodulatory activity, and it is called host defense peptide (HDP) [12,13]. Unfortunately, very limited information is available on E. necatrix and AMPs/HDPs that could kill Eimeria spp. and/or modulate the immune response in chickens. cNK- 
lysin is the first host-derived peptide reported anticoccidial activity. Thus, this study aimed to investigate the expression of cNK-lysin in E. necatrix-infected chickens and its role in E. necatrix infection.

\section{MATERIALS AND METHODS}

\section{Chickens and parasites}

A total of 40 broiler chickens (Ross/Ross) were used in this study and all birds were raised in petersime battery cages. Feed and water were provided ad libitum under coccidian-free conditions. The sporozoites of E. necatrix (GNU strain) were obtained by excystation of sporulated oocysts [11]. Briefly, freshly sporulated oocysts were disrupted with 0.5 -mm glass beads for 10-15 sec by using a Mini-beadbeater (BioSpec Products, Bartlesville, Oklahoma, USA). The released sporocysts were purified by isopycnic centrifugation in a Percoll gradient and washed in Hank's balanced salt solution (HBSS, MilliporeSigma), and the excystation of sporozoites was conducted by treatment with $0.25 \%$ trypsin and $0.014 \mathrm{M}$ taurocholic acid (all from MilliporeSigma) at $41^{\circ} \mathrm{C}$ for $90 \mathrm{~min}$. The excysted sporozoites were collected, washed 3 times with HBSS at 3,000 $\times \mathrm{g}$ for 10 $\min$ at $4^{\circ} \mathrm{C}$ and used at the same day of experiment. All animal maintenance and experimental procedures were performed in accordance with Gyeongsang National University Guidelines for the Care and Use of Experimental Animals and were approved by the Institutional Animal Care and Use Committee (IACUC) of Gyeongsang National University (GNU-191111C0058).

\section{Lymphocytes preparation and cell culture}

Chicken intestinal epithelial lymphocytes (IELs) and splenic mononuclear cells (SMCs) were isolated from jejunum and spleen, respectively, as previously described with modification $[6,14]$. Briefly, jejunum was collected aseptically from healthy or $E$. necatrix-infected chicken and washed with ice-cold $\mathrm{Ca}^{+}{ }^{+}$ and Mg2 ${ }^{+}$-free HBSS (CMF-HBSS, GE Healthcare, Pittsburgh, Pennsylvania, USA) containing $1 \mathrm{mM}$ dithiothreitol (MilliporeSigma, St. Louis, Missouri, USA) followed by incubation with CMF-HBSS containing $0.5 \mathrm{mM}$ EDTA and 5\% FBS (GE Healthcare) for $20 \mathrm{~min}$ at $37^{\circ} \mathrm{C}$ with constant swirling. Cells released into the supernatant were pooled, passed through nylon wool (Poly Sciences, Warrington, Pennsylvania, USA), and purified by a Histopaque-1077(MilliporeSigma) density gradient method. For SMCs, collected spleen was homogenized us- ing gentleMACS Dissociator (Miltenyi Biotec, Gaithersburg, USA) and purified by the same method described above. Freshly purified lymphocytes were cultured in complete RPMI1640 (GE Healthcare) supplemented with 10\% FBS, penicillin/streptomycin (10,000 unit/ml, Invitrogen, Carlsbad, California, USA), $50 \mu \mathrm{g} / \mathrm{ml}$ gentamycin, $25 \mathrm{mM}$ HEPES, and 55 $\mu \mathrm{M}$ 2-Mercaptoethanol (all from MilliporeSigma). The chicken macrophage cell line HD11 was maintained in complete DMEM supplemented the same as RPMI-1640 described above.

\section{E. necatrix infection and real-time $\mathrm{qPCR}$}

Total 40 chickens were divided by 2 groups, infected and non-infected. The infected group was orally infected with $E$. necatrix sporulated oocysts $\left(1 \times 10^{4} / \mathrm{ml} / \mathrm{bird}\right)$ at 14 days old and the non-infected group was given HBSS as a control. The jejunal tissues and spleen were collected from 5 chickens of each group at 1, 4, 7, and 10 days post infection (DPI) to isolate IELs and SMCs, respectively. For mRNA expression analysis, RNA was isolated by using a RNeasy Isolation Kit (Qiagen, Germantown, Maryland, USA) then treated with RNase-free DNase (Qiagen) and eluted in RNase-free water (Qiagen). The concentration and purity of the RNA were measured using a NanoDrop spectrophotometer (Thermo Fisher Scientific, Frederick, Maryland, USA). cDNA was synthesized using random hexamer primers and a QuantiTect Reverse Transcription Kit (Qiagen). Real-time qPCR was performed using a CFX96 realtime PCR system (Bio-Rad, Hercules, California, USA) with a QuantiTect SYBR Green PCR Kit (Qiagen). The primers sequences for chicken $\beta$-actin and cNK-lysin are list in Table 1. The fold changes in each transcript were normalized to $\beta$-actin.

Table 1. List of real-time q-PCR primers used in this study

\begin{tabular}{|c|c|c|}
\hline Target & Primer and sequence & Reference \\
\hline cNK-lysin & $\begin{array}{l}\text { (For) 5'-GATGGTTCAGCTGCGTGGGATGC-3' } \\
\text { (Rev) 5'-CTGCCGGAGCTTCTTCAACA-3' }\end{array}$ & [7] \\
\hline $\mathrm{IL}-1 \beta$ & $\begin{array}{l}\text { (For) 5'-TGGGCATCAAGGGCTACA-3' } \\
\text { (Rev) 5'-TCGGGTTGGTTGGTGATG-3' }\end{array}$ & [11] \\
\hline CXCLi2 & $\begin{array}{l}\text { (For) 5'-GGCTTGCTAGGGGAAATGA-3' } \\
\text { (Rev) 5'-AGCTGACTCTGACTAGGAAACTGT-3' }\end{array}$ & [11] \\
\hline CCL4 & $\begin{array}{l}\text { (For) 5'-GCTGCCCTTCAGCTTTG-3' } \\
\text { (Rev) 5'-TCAGTTCAGTTCCATCTTGTTCATGTA-3' }\end{array}$ & [11] \\
\hline CCL5 & $\begin{array}{l}\text { (For) 5'-TATTTCTACACCAGCAGCAAATG-3' } \\
\text { (Rev) 5'-GCAGACACCTCAGGTCC-3' }\end{array}$ & {$[11]$} \\
\hline$\beta$-actin & $\begin{array}{l}\text { (For) 5'-CACAGATCATGTTTGAGACCTT-3' } \\
\text { (Rev) 5'-CATCACAATACCAGTGGTACG-3' }\end{array}$ & [11] \\
\hline
\end{tabular}




\section{Recombinant cNK-lysin expression}

The recombinant cNK-lysin (rcNK-lysin) protein was obtained from pcDNA3.1-cNK-lysin transfected COS-7 cells (ATCC, Manassas, Virginia, USA). Briefly, full-length cNK-lysin CDNA was amplified from E. necatrix-infected ceca by PCR using the following primers (5'-GATCAAGCTT ATGGCCGCTGCTCTCATC-3' and 5'GATCGAATTCTCAGCCCCTTGCCAGCCC-3'). Primers contained HindIII and EcoRI restriction enzyme sites (single underlined). PCR products were digested with HindIII and EcoRI and cloned into the corresponding restriction sites of pcDNA3.1 (Invitrogen). The COS-7 cells were transiently transfected with $10 \mu \mathrm{g}$ of constructs or empty vector (negative control) using FuGENE (Promega, Madison, Wisconsin, USA) according to the manufacturer's instructions. The supernatants containing rcNK-lysin were collected after $48 \mathrm{hr}$ of transfection.

\section{Western blot}

Collected supernatants from COS-7 cells were concentrated about 20-fold by using Amicon Ultra Centrifugal Filter Unit (MilliporeSigma). Concentrated samples were mixed with an equal volume of sample buffer (Bio-Rad), heated for $5 \mathrm{~min}$ at $95^{\circ} \mathrm{C}$, resolved on a TGX Precast gel (Bio-Rad) and electroblotted onto polyvinyl difluoride membranes using a Trans-Blot Transfer System (Bio-Rad). The membranes were blocked with PBS SuperBlock (Thermo Fisher Scientific) Blocking Buffer for $1 \mathrm{hr}$, washed with $0.1 \%$ Tween 20 in PBS (PBS-T), and probed with anti-serum (1:100) obtained from rabbits immunized with cNK-2, cNK-lysin-derived peptide (aa101-127, RRQRSICKQLLKKLRQQLSDALQNNDD), followed by HRP-conjugated goat anti-rabbit IgG (Thermo Fisher Scientific). The membranes were washed 5 times with PBS-T, visualized using a Clarity ECL Western Blotting Substrate (Bio-Rad), and detected using the ChemiDoc Imaging System (Bio-Rad).

\section{E. necatrix killing assay}

To test anticoccidial effect of rcNK-lysin in vitro, isolated $E$. necatrix sporozoites was stained with Carboxyfluorescein succinimidyl ester (CFSE, abcam) according to the manufacturer's instructions. Then the collected sporozoites were incubated at $41^{\circ} \mathrm{C}$ with supernatant containing rcNK-lysin for $3 \mathrm{hr}$. The viability of sporozoites was measure in triplicate by using a Synergy HTX Plate reader (BioTek, Winooski, VT) with a 485/528 filter. The viability of sporozoites showed linear correlation with fluorescence intensity and it was presented as percentage of $\{($ sample intensity/media control intensity $) \times 100\}$.

\section{Statistical analysis}

The data were analyzed using Student's t-test or one-way ANOVA followed by Dunnett's multiple comparison test (Prism Version 5.01, GraphPad Software, La Jolla, California, USA). Differences were considered significant at $P<0.05$ or $P<0.01$. The data are expressed as the mean value \pm the standard error (SE).

\section{RESULTS}

\section{Increased cNK-lysin expression in E. necatrix-infected chicken lymphocytes}

To investigate if E. necatrix could induce cNK-lysin expression, primary lymphocytes were prepared from jejunum (IELs) which is the site of $E$. necatrix invasion and spleen (SMCs). After the stimulation with live E. necatrix sporozoites, which are an invasive stage of parasites, both lymphocytes exhibited significantly increased expression of cNK-lysin up to $8 \mathrm{hr}$ but this induction did not last for $24 \mathrm{hr}$. When the lymphocytes were stimulated with $E$. necatrix sporocysts, preceding the stage of sporozoites released in the intestine, the expression of cNK-lysin was remained unchanged. There was no remarkable difference in expression pattern between IELs and SMCs but interestingly, the amounts of induced transcript in SMCs were more than those in IELs (Fig. 1). The E. necatix oocysts were also tested on the same cells preliminarily but no changes in cNK-lysin expression were found (data not shown).

\section{mRNA expression of cNK-lysin in E. necatrix-infected chickens}

To validate in vitro finding that $E$. necatrix induces cNK-lysin expression, 2 weeks old broilers were infected with E. necatrix oocysts and the expression of cNK-lysin was analyzed in Eimeria infected lymphocytes isolated from infected chickens. In agreement with in vitro findings, E. necatrix infection increased cNK-lysin expression significantly in IELs. At 4 DPI, it showed the highest expression ( $\sim 45.1$ fold) followed by 7 DPI ( 7.6 fold) compared to non-infected chickens (Fig. 2A). Unlike IELs, SMCs showed a slight increase at 4 DPI ( 3.1 fold) or unchanged in cNK-lysin expression (Fig. 2B). This discrepancy between in vitro and in vivo experiments indicates that the cNKlysin expression induced by E. necatrix is site-specific and cNKlysin plays a role exclusively in the local intestine. 

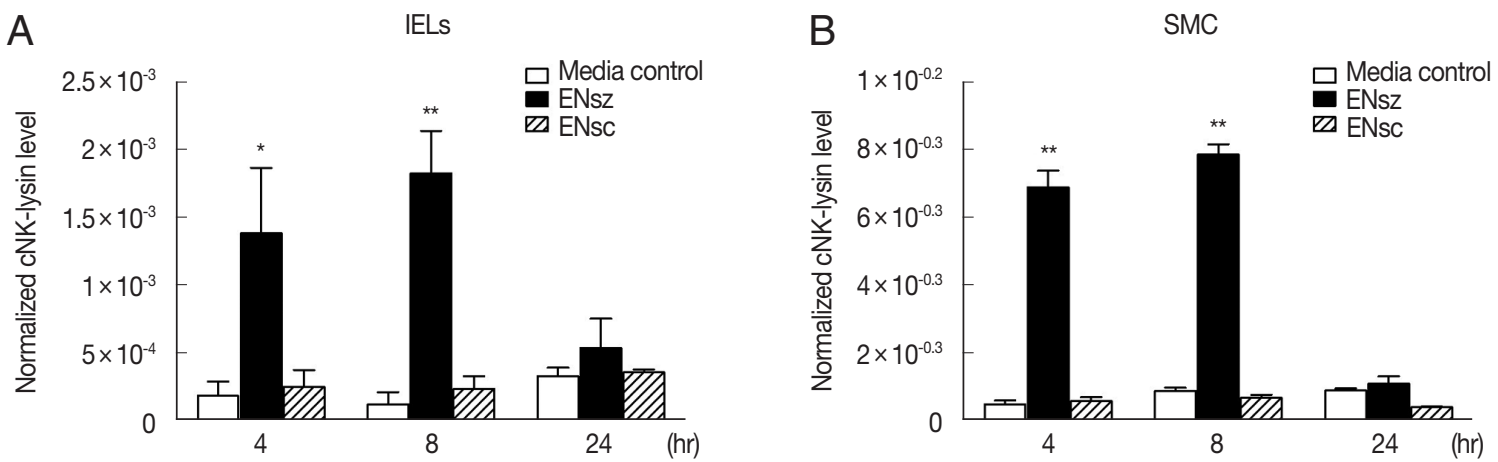

Fig. 1. Expression of cNK-lysin transcript in chicken lymphocytes infected with Eimeria necatrix. Chicken lymphocytes were isolated from healthy birds and stimulated with $1 \mu \mathrm{g} / \mathrm{ml}$ E. necatrix sporozoites or sporocysts protein. The mRNA expression of cNK-lysin in the jejunal intraepithelial lymphocytes (A) and splenic mononuclear cells (B) by real-time qPCR and normalized to $\beta$-actin. The data represent the average of 3 independent experiments \pm SE. $P<0.05\left(^{*}\right)$ and $P<0.01\left(^{(*)}\right.$ were considered statistically significant compared to the respective media control. ENsz, E. necatrix sporozoites; ENsc, E. necatrix sporocysts.
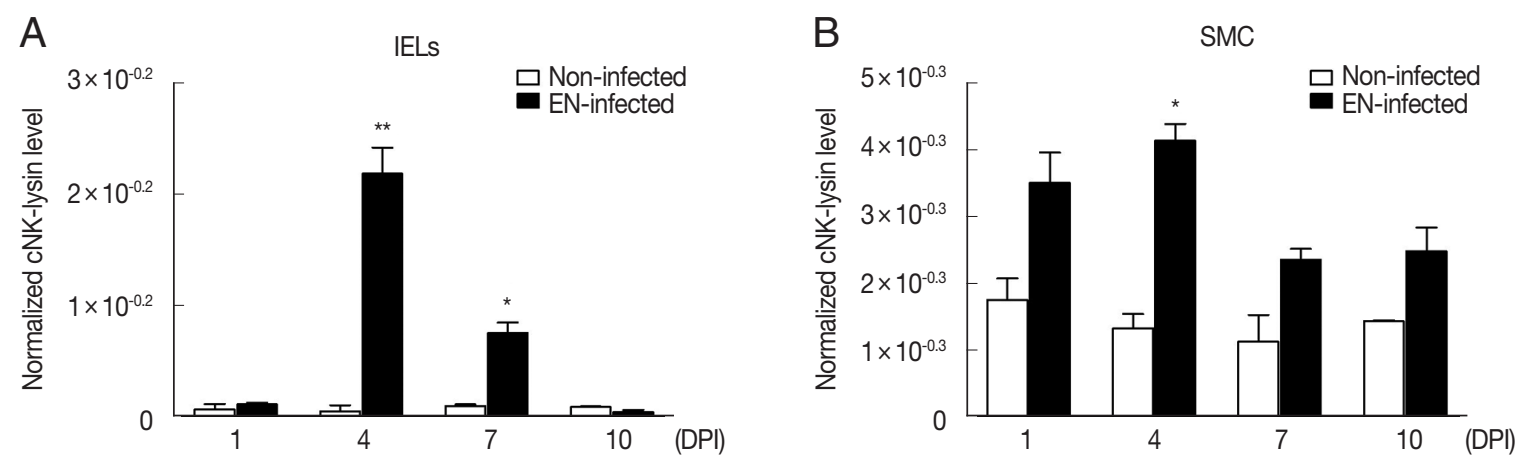

Fig. 2. Expression of cNK-lysin transcript in Eimeria necatrix-infected chickens. Two-weeks-old chickens were given $1 \times 10^{4}$ E. necatrix oocysts and the mRNA expression of cNK-lysin was analyzed in jejunal intraepithelial lymphocytes (A) and splenic mononuclear cells (B) by real-time qPCR and normalized to $\beta$-actin. The data represent the average of 2 independent experiments \pm SE. $P<0.05\left(^{*}\right)$ and $\left.P<0.01{ }^{(\star}\right)$ were considered statistically significant compared to the respective non-infected control $(n=5)$. EN, E. necatrix; DPI, days post-infection.

\section{Direct effects of cNK-lysin on E. necatrix}

As cNK-lysin has been reported as an anticoccidial peptide, cNK-lysin expressed from the mammalian system was next used to see if it has antimicrobial activity against $E$. necatrix. The expression of cNK-lysin was confirmed by using an anticNK-2 antibody. Western blot analysis presented that cNK-lysin protein was successfully expressed and the observed band was between 10 and $15 \mathrm{kDa}$ in molecular weight (MW) (Fig. 3A). Based on the amino acid sequence, the predicted MW for cNK-lysin is $15.2 \mathrm{kDa}$. Due to the limitation on protein yield in COS-7 cells, the supernatants were collected and serially diluted with growth medium before the treatment. As expected, the supernatant containing cNK-lysin exhibited antimicrobial activity in a dose-dependent manner which is consistent with a previous report using other species of Eimeria in chickens $[9,10]$ (Fig. 3B). In the assay to test E. necatrix sporocysts, via- bilities were unchanged indicating that cNK-lysin could not affect the wall of sporocysts (Fig. 3C).

\section{Effects of cNK-lysin in induction of cytokines and chemokines on macrophages}

The previous study has reported that in some parts of cNKlysin, cNK-2 has immunomodulatory properties, including induction of cytokines and chemokines, suppression of inflammatory response, and signaling pathway activation in chicken macrophages [11]. Prior to investigating the effect of cNK-lysin in cytokine and chemokine induction on macrophages, jejunal IELs were prepared from healthy chickens and infected with $E$. necatrix to obtain E. necatrix-induced native cNK-lysin. The stimulation of the supernatant containing cNK-lysin collected from IELs culture significantly induced the expression of inflammatory cytokines, interleukin (IL)-1 $\beta$ and CXCLi2, one 

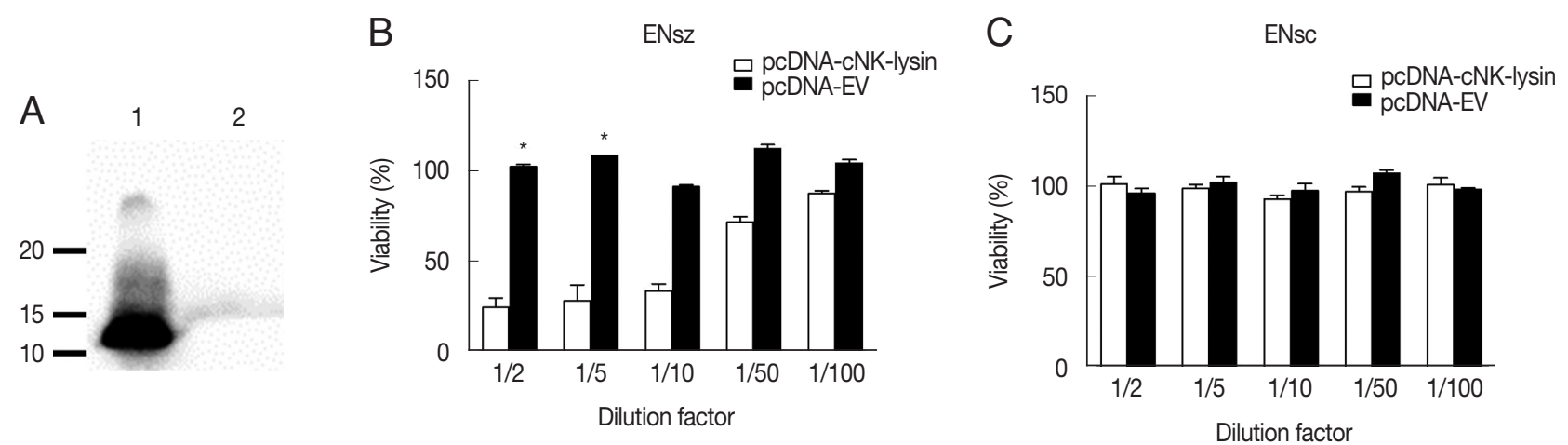

Fig. 3. Antimicrobial activity of cNK-lysin against Eimeria necatrix. (A) Detection of cNK-lysin protein. Concentrated supernatants of pcDNA-cNK-Iysin (lane 1) or pcDNA-EV (lane 2) transfected COS-7 cells were blotted with anti-cNK-2 rabbit serum. The antimicrobial activity of cNK-lysin-containing supernatant against $E$. necatrix sporozoites (B) and sporocysts (C) was determined. The viability was calculated relative to non-treated media control sample. The data represent the average of 2independent experiments $\pm S E . P<0.05\left({ }^{*}\right)$ was considered statistically significant compared to the respective media control. EV, empty vector.
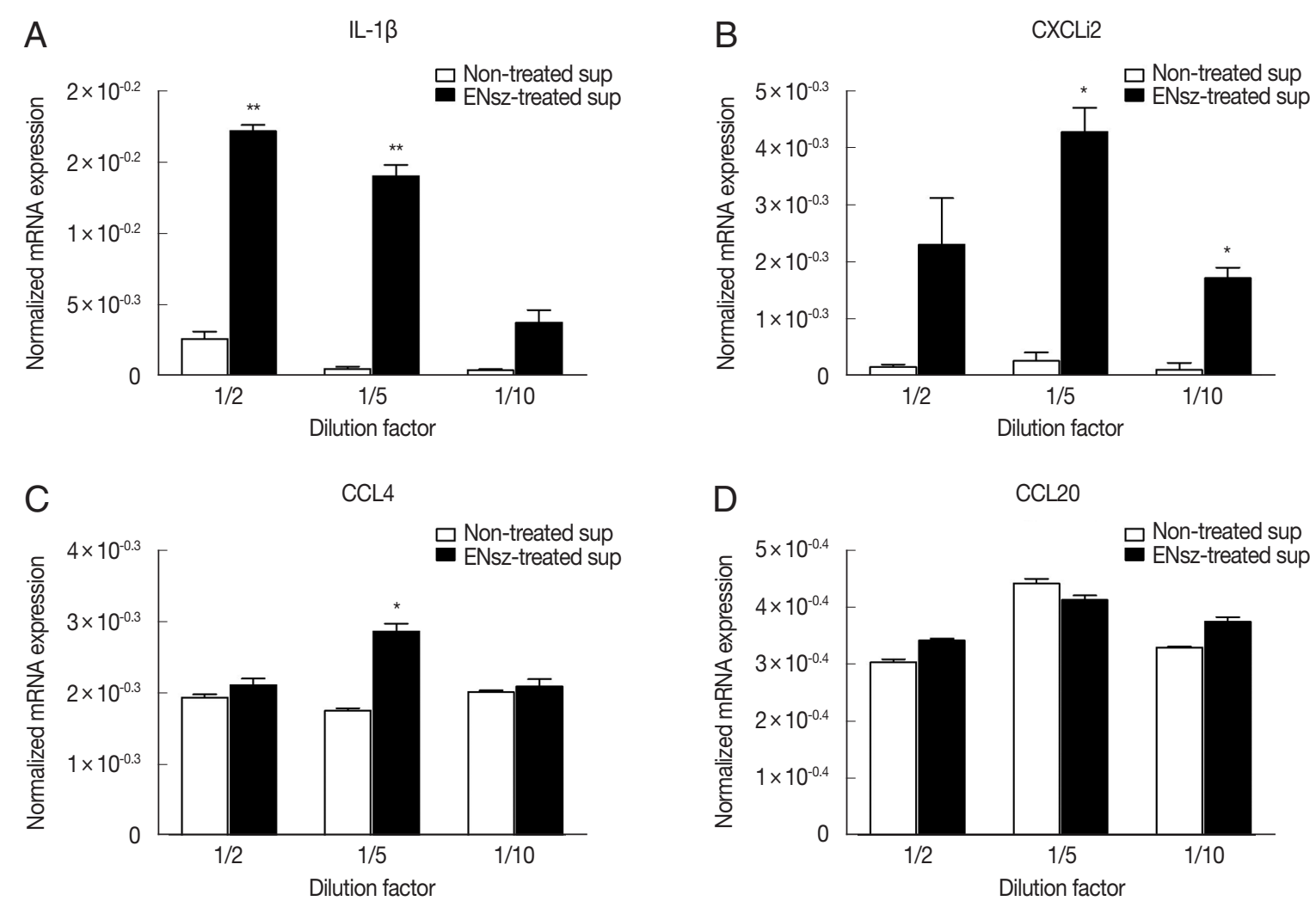

Fig. 4. Immunomodulatory effect of cNK-lysin on chicken macrophages. IELs isolated from healthy chickens were infected with E. necatrix for $4 \mathrm{hr}$. HD11 cells were stimulated with supernatants collected from E. necatrix sporozoites-treated jejunal intraepithelial cells. The mRNA expressions of IL-1 $\beta$ (A), CXCLi2 (B), CCL4 (C), and CCL20 (D) were analyzed by real-time qPCR and normalized to $\beta$-actin. The data represent the average of 2 independent experiments \pm SE. $P<0.05\left(^{*}\right)$ and $P<0.01\left(^{(\star}\right)$ were considered statistically significant compared to the respective non-treated supernatant control. ENsz, E. necatrix sporozoites.

of chicken homolog of CXCL8 on HD11 (Fig. 4A, B). In contrast to the previous finding, CCL4 and CCL20 which CNK-2 in previous study induced were not increased by the stimulation of cNK-lysin-containing supernatant compared to non- treated supernatant (Fig. 4C, D). This result suggested that cNK-lysin and cNK-2 might act differently in chicken macrophages, although cNK-lysin contains exactly the same sequence of cNK-2. 


\section{DISCUSSION}

The interest in the role of cNK-lysin in chicken coccidiosis has begun from an initial study that reported a huge number of the expressed sequence tags in E. acervulina and E. maxima infection. Min et al. [6], constructed cDNA library of Eimeriainfected IELs and found the highest expression of an unknown gene with high homology with bovine NK-lysin. Later, a chicken homolog has cloned, and it has shown to express in IELs and have antimicrobial activity against Eimeria spp. including E. acervulina, E. maxima, and E. tenella $[7,9,10]$. However, no studies are conducted using other Eimeria species like E. necatrix which is the one of the most pathogenic species resulting in hemorrhagic lesion in the mid-intestine with a high mortality [2]. In this study, it was found that E. necatrix could induce cNK-lysin expression in lymphocytes. Interestingly, its expression has shown in IELs and SMCs after the treatment of E. necatrix sporozoites, whereas IELs, but not SMCs expressed cNKlysin in E. necatrix-infected chicken. This suggests that lymphocytes maybe cytotoxic $\mathrm{T}$ and $\mathrm{NK}$ cells, are the responder cells expressing cNK-lysin and IELs are the most cells responding to sporozoites in E. necatrix-infected chickens as SMCs did not express cNK-lysin as much as IELs. Limited expression of cNKlysin in the intestine is supported by studies emphasizing the importance of intestinal immunity in the prevention of coccidiosis [15-18]. The result showing highly induced cNK-lysin by $E$. necatrix leads to investigation on the role of cNK-lysin in coccidiosis. The following study showed that rcNK-lysin expressed from COS-7 cells has antimicrobial activity against $E$. necatrix sporozoites and that $\mathrm{CNK}$-lysin containing supernatant upregulates proinflammatory cytokine expression in macrophages. Similar to that $E$. necatrix sporocoysts did not induce cNK-lysin, the only sporozoites could be a target lysed by cNKlysin. Throughout this study, in coccidiosis, a developmental stage that elicits an immune response is the sporozoites not the sporocysts. Sporozoites are motile and able to actively invade the primarily intestinal epithelium. Macrophages and IELs have also been reported as the target cells of sporozoites but IELs, unlike intestinal epithelial cells are believed to be responsible for sporozoites transport rather than parasite development. Especially, E. necatrix, E. acervulina, E. maxima and E. tenella are the species that transport sporozoites to other sites, for example, the crypt [19-23]. In a previous study, cNK-2 has been reported to induce chemokines and to suppress inflammatory response [11]. However, in this study, native cNK-lysin induced proinflammatory cytokines, IL-1 $\beta$, and CXCLi2. It is not clear whether cNK-lysin is related to inflammatory or antiinflammatory, but it seems cNK-lysin participates in E. necatrixinduced immune response somehow. Another thing that is distinct to $\mathrm{CNK}-2$ was the chemokine induction as cNK-lysin did not induce CCL4 and CCL20 whereas they are highly induced by cNK-2 which suggests a different role of cNK-lysin. The differences from the previous report could come from the species of Eimeria, and further study on detailed properties of each species will be needed. In summary, so far, very few AMPs showing anticoccidial property have been studied although a huge effort has been made in the development of AMPs targeting bacterial pathogens. Thus, this study provides the knowledge to use AMPs to prevent coccidiosis and understand the immune response in coccidia infection. Further studies will be required to elucidate the exact role of cNK-lysin in coccidiosis.

\section{ACKNOWLEDGMENT}

This work was supported by IPET through the Animal Disease Management Technology Development Program, funded by MAFRA (320062-2).

\section{CONFLICT OF INTEREST}

The authors declare that there are no known competing financial interests or personal relationships that could have appeared to influence the work reported in this paper.

\section{REFERENCES}

1. Shirley MW, Lillehoj HS. The long view: a selective review of 40 years of coccidiosis research, Avian Pathol 2018; 41: 111-121. https://doi.org/10.1080/03079457.2012.666338

2. Taylor MA, Coop RL, Wall RL. Veterinary Parasitology, 3rd ed. Oxford, UK. Blackwell Publishing, 2007.

3. Haug A, Gjevre AG, Thebo P, Mattsson JG, Kaldhusdal M. Coccidial infections in commercial broilers: Epidemiological aspects and comparison of Eimeria species identification by morphometric and polymerase chain reaction techniques, Avian Pathol 2008; 37: 161-170. https://doi.org/10.1080/03079450801915130

4. Blake DP, Pastor-Fernández I, Nolan MJ, Tomley FM. Recombinant anticoccidial vaccines-a cup half full? Infect Genet Evol 2017; 55: 358-365. https://doi.org/10.1016/j.meegid.2017.10.009

5. Venkatas J, Adeleke MA. A review of Eimeria antigen identification for the development of novel anticoccidial vaccines, Parasitol Res 2019; 118: 1701-1710. https://doi.org/10.1007/s00436-019-06338- 
2

6. Min W, Lillehoj HS, Ashwell CM, Van Tassell CP, Dalloul RA, Matukumalli LK, Han JY, Lillehoj EP. Expressed sequence tag analysis of Eimeria-stimulated intestinal intraepithelial lymphocytes in chickens. Mol Biotechnol 2005; 30; 143-150. https:// doi.org/10.1385/MB:30:2:143

7. Hong YH, Lillehoj HS, Dalloul RA, Min W, Miska KB, Tuo W, Lee SH, Han JY, Lillehoj EP. Molecular cloning and characterization of chicken NK-lysin, Vet Immunol Immunopathol 2006; 110: 339-347. https://doi.org/10.1016/j.vetimm.2005.11.002

8. Kim WH, Lillehoj HS, Gay CG. Using genomics to identify novel antimicrobials. Rev Sci Tech 2016; 35: 95-103. https://doi.org/ 10.20506/rst.35.1.2420

9. Hong YH, Lillehoj HS, Siragusa GR, Bannerman DD, Lillehoj EP. Antimicrobial activity of chicken NK-Lysin against Eimeria sporozoites. Avian Dis 2008; 52: 302-305. https://doi.org/10.1637/ 8083-072307-resnote.1

10. Lee SH, Lillehoj HS, Tuo W, Murphy CA, Hong YH, Lillehoj EP. Parasiticidal activity of a novel synthetic peptide from the core a-helical region of NK-lysin. Vet Parasitol 2013; 197: 113-121. https://doi.org/10.1016/j.vetpar.2013.04.020

11. Kim W, Lillehoj HS, Min W. Evaluation of the immunomodulatory activity of the chicken NK-Lysin-Derived peptide cNK-2. Sci Rep 2017; 7: 45099 https://doi.org/10.1038/srep45099

12. Haney EF, Hancock REW. Peptide design for antimicrobial and immunomodulatory applications. Biopolymers 2013; 100: 572 583. https://doi.org/10.1002/bip.22250

13. Haney EF, Straus SK, Hancock REW. Reassessing the host defense peptide landscape. Front Chem 2019; 7: 1-22. https://doi.org/ 10.3389/fchem.2019.00043

14. Kim WH, Jeong J, Park AR, Yim D, Kim S, Chang HH, Yang SH, Kim DH, Lillehoj HS, Min W. Downregulation of chicken interleukin-17 receptor A during Eimeria infection. Infect Immun
2014; 82: 3845-3854. https://doi.org/10.1128/IAI.02141-14

15. Lillehoj HS, Trout JM. Avian gut-associated lymphoid tissues and intestinal immune responses to Eimeria parasites. Clin Microbiol Rev 1996; 9: 349-360. https://doi.org/10.1128/cmr.9.3.349

16. Lillehoj HS, Lillehoj EP. Avian Coccidiosis. A review of acquired intestinal immunity and vaccination strategies. Avian Dis 2000; 44: 408-425. https://www.jstor.org/stable/1592556

17. Yun $\mathrm{CH}$, Lillehoj HS, Choi KD. Eimeria tenella infection induces local gamma interferon production and intestinal lymphocyte subpopulation changes. Infect Immun 2000; 63: 1282-1288. https://doi.org/10.1128/IAI.68.3.1282-1288.2000

18. Yun $\mathrm{CH}$, Lillehoj HS, Lillehoj EP. Intestinal immune responses to coccidiosis. Dev Comp Immunol 2000; 24: 303-324. https:// doi.org/10.1016/S0145-305X(99)00080-4

19. Al-Attar MA, Fernando MA. Transport of Eimeria necatrix sporozoites in the chicken: effects of irritants injected intraperitoneally. J Parasitol 1987; 73: 494-502. https://www.jstor.org/stable/ 3282127

20. Fernando MA, Elaine Rose M, Millard BJ. Eimeria spp. of domestic Fowl: the migration of sporozoites intra-and extra-enterically. J Parasitol 1987; 73: 561-567. https://www.jstor.org/stable/3282137

21. Lawn AM, Rose ME. Mucosal transport of Eimeria tenella in the cecum of the chicken. J Parasitol 1982; 68: 1117-1123. https://www. jstor.org/stable/3281101

22. Trout JM, Lillehoi HS. Evidence of a role for intestinal CD8+ lymphocytes and macrophages in transport of Eimeria acervulina sporozoites. J Parasitol 1993; 79: 790-792. https://www.jstor.org/ stable/3283625

23. Trout JM, Lillehoj HS. Eimeria acervulina infection: evidence for the involvement of CD8+ Tlymphocytes in sporozoite transport and host protection. Poult Sci 1995; 74: 1117-1125. https://doi. org/10.3382/ps.0741117 
